\title{
Perfilhamento e expansão de touceiras de helicônias ${ }^{1}$
}

\author{
Andreza S da Costa ${ }^{2}$; Vivian Loges ${ }^{3}$; Ana Cecília R de Castro ${ }^{4}$; André Luiz Verona ${ }^{5}$; Cleucione de O \\ Pessoa $^{5}$; Venézio Felipe dos Santos ${ }^{6}$ \\ ${ }^{2}$ UFRPE-PPGMGP, Av. D. Manoel de Medeiros, s/n ${ }^{\circ}, 52171-900$ Recife-PE; ${ }^{3}$ UFRPE-DEPA-Área de Fitotecnia; ${ }^{4}$ EMBRAPA-CNPAT,

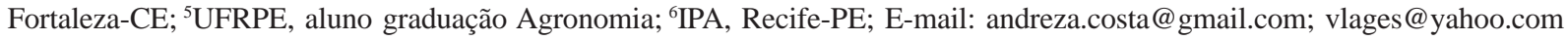

\section{RESUMO}

Neste trabalho foram avaliados, para o espaçamento $1,5 \times 3,0$ m, o número de perfilhos por touceira (NPT) e a área de ocupação da touceira (AOT) de 26 genótipos de Heliconia spp., cultivados a pleno sol e a meia-sombra. O delineamento experimental foi blocos ao acaso, com quatro repetições. $\mathrm{Na}$ avaliação realizada 316 dias após o plantio (DAP), os genótipos cultivados a pleno sol apresentaram médias de NPT variando entre 18,3 (H. bihai cultivar Kamehameha) e 57,0 (H. psittacorum cultivar Red Opal), agrupando-se em três classes distintas (Scott-Knott, $\mathrm{P}<0,05$ ). Os genótipos cultivados a meia-sombra apresentaram NPT médio variando entre 11,7 (H. bihai cultivar Nappi Yellow) e 43,7 (H. psittacorum x H. spathocircinata cultivar Golden Torch), agrupando-se em três classes distintas (Scott-Knott, $\mathrm{P}<0,05$ ). De modo geral, observou-se que as helicônias de pequeno porte como, por exemplo, as cultivares e híbridas de $H$. psittacorum perfilharam mais que as helicônias de grande porte, em ambas as condições de plantio. Em relação à AOT, na avaliação realizada 304 DAP entre os materiais cultivados a pleno sol, $H$. psittacorum cultivar Red Opal ocupou apenas metade da área da parcela $\left(11.387 \mathrm{~cm}^{2}\right)$, enquanto o genótipo Heliconia $\mathrm{x}$ nickeriensis ocupou toda a área $\left(22.541 \mathrm{~cm}^{2}\right)$. $\mathrm{O}$ genótipo $H$. orthotricha apresentou a menor AOT $\left(725 \mathrm{~cm}^{2}\right)$. Para os genótipos cultivados a meia-sombra, a AOT variou entre $750 \mathrm{~cm}^{2}(H$. bihai cutivar Nappi Yellow) e $10.201 \mathrm{~cm}^{2}$ (H. stricta cultivar Fire Bird). Embora as avaliações continuem em andamento, os resultados obtidos até 316 DAP permitem concluir que a adoção de um mesmo espaçamento para diferentes genótipos de helicônia é inadequada e que poderá acarretar futuros problemas de manejo das plantas.

Palavras-chave: Heliconia spp., manejo cultural, espaçamento, caracterização.

\begin{abstract}
Number of shoots and area per clump of heliconia

This paper presents information on the number of shoots per clump (NSC) and area per clump (APC) of 26 Heliconia spp. genotypes, cultivated in full sun and partial shade, at a plant spacing of 3,0 x 1,5 m. 316 days after planting (DAP), NSC of genotypes grown in full sun varied from 18.3 (H. bihai cultivar 'Kamehameha') to 57.0 (H. psittacorum cultivar 'Red Opal'), splitting in three distinct classes (Scott-Knott, $\mathrm{P}<0,05)$. For genotypes grown in partial shade, NSC varied from 11.7 (H. bihai cultivar 'Nappi Yellow') to 43.7 ( $H$. psittacorum $x$ H. spathocircinata cultivar 'Golden Torch'), splitting also in three distinct classes (Scott-Knott, $\mathrm{P}<0,05)$. It was observed that short heliconia genotypes, as the cultivars and hybrids of $H$. psittacorum, have a larger number of shoots than tall heliconia genotypes, in both planting conditions. Results from the APC, measured 304 DAP, showed that among the genotypes grown in full sun, $H$. psittacorum cultivar 'Red Opal' occupied half of the plot area $\left(11.387 \mathrm{~cm}^{2}\right)$ while genotype Heliconia $x$ nickeriensis occupied an area larger than the plot area $\left(22.541 \mathrm{~cm}^{2}\right)$. The genotype $H$. orthotricha presented the smallest APC $\left(725 \mathrm{~cm}^{2}\right)$. In shaded condition APC varied from $750 \mathrm{~cm}^{2}$ (H. bihai 'Nappi Yellow') to $10.201 \mathrm{~cm}^{2}$ (H. stricta 'Fire Bird'). The evaluation of these traits during the first 316 DAP allowed us to conclude that a single plant spacing for different heliconia genotypes is inappropriate and can lead to future problems in plant management.
\end{abstract}

Keywords: Heliconia spp., crop management, spacing, characterization.

(Recebido para publicação em 22 de fevereiro de 2006; aceito em 19 de dezembro de 2006)

$\mathrm{O}$ agronegócio da floricultura movimenta anualmente no mercado mundial aproximadamente US\$ 94 bilhões, mas com uma demanda que é aproximadamente o dobro da atual ( $\mathrm{Ri}$ beiro et al., 2002). Em 2003, o comércio exterior brasileiro de produtos ligados à floricultura teve um desempenho excepcional, com um aumento de $30 \%$ no valor exportado em relação ao ano anterior, atingindo o patamar inédito de US\$ 20 milhões (Kiyuna et al., 2004). Em relação à floricultura tropical,
Pernambuco destaca-se como um dos maiores produtores de helicônias (Heliconia spp.), apresentando aumento da área plantada e das exportações nos últimos anos (Aki \& Perosa, 2002; Junqueira \& Peetz, 2002).

As helicônias são plantas herbáceas, perenes, rizomatosas, formando touceiras de população monoclonal com emissão de perfilhos (Criley \& Broschat, 1992) que se desenvolvem principalmente na periferia da touceira (Criley, 1989). A variação do perfilhamento em helicônia é associada a características genéticas, mas também influenciada por outros fatores como condições climáticas (Geertsen, 1989; Fernandes, 2000). Para a cultivar Golden Torch, a faixa de temperatura ótima para produção de perfilhos foi de 22-26 ${ }^{\circ} \mathrm{C}$ (Catley \& Brooking, 1996).

O espaçamento de cultivo para as helicônias é definido pelo hábito de crescimento, que pode ser agrupado ou aberto. Plantas com crescimento agrupado desenvolvem-se lentamente e com has-

\footnotetext{
${ }^{1}$. Parte da dissertação da primeira autora apresentada ao Programa de Pós-Graduação em Melhoramento Genético de Plantas da Universidade Federal Rural de Pernambuco (UFRPE)
} 
tes verticais, formando touceiras mais fechadas. Espécies com crescimento aberto apresentam desenvolvimento rápido e touceiras com arquitetura dispersa necessitando de maior espaçamento (Criley, 1988). O conhecimento da área de ocupação da touceira ao longo do tempo é importante por estar diretamente relacionado ao espaçamento de plantio e manejo da cultura.

O espaçamento deve variar de acordo com a espécie (Atehortua, 1998). Para espécies produtoras de flores pesadas, eretas ou pendentes, e que formam touceiras grandes, com plantas acima de 1,5 m de altura, deve ser mantido um espaçamento de $0,8 \times 0,8 \mathrm{~m}$ ou superior, em canteiros distanciados entre si por 1,0 ou 1,5 m (Castro, 1995). Segundo Paiva (1998), são utilizados espaçamento de 1,50 x $0,80 \mathrm{~m}$ para helicônias de porte médio a grande e de $1,20 \times 0,20 \mathrm{~m}$ para helicônias de porte pequeno. No entanto, de acordo com Ibiapaba et al. (1997), o espaçamento que apresentou o melhor resultado para produção em $H$. stricta e $H$. rauliniana, que são plantas de grande porte, foi de $0,5 \mathrm{x}$ $1,0 \mathrm{~m}$. Alguns produtores na Zona da Mata de Pernambuco têm adotado espaçamento de $3,0 \times 3,0 \mathrm{~m}$ e $3,0 \times 4,0 \mathrm{~m}$.

O objetivo deste trabalho foi avaliar o número de perfilhos e a área de ocupação da touceira de 26 genótipos de helicônia (Tabela 1). Essas são as informações necessárias para identificar o espaçamento mais adequado para cada genótipo.

\section{MATERIAL E MÉTODOS}

O experimento foi implantado em dezembro de 2003, no município de Camaragibe-PE, situado a $8^{\circ} 1$ ' 19 " de latitude sul, 34 $59^{\prime} 33^{\prime}$ de longitude oeste e a $100 \mathrm{~m}$ de altitude. A temperatura média da região é de $25,1^{\circ} \mathrm{C}$, precipitação média mensal de $171 \mathrm{~mm}$, com máxima de $377 \mathrm{~mm}$ e mínima de $37 \mathrm{~mm}$ (histórico de sete anos, ITEP, 2005).

$\mathrm{Na}$ área experimental, que possui solo franco argiloso classificado como latossolo vermelho-amarelo, foi feita calagem com calcário dolomítico, seis meses antes do plantio. A adubação foi realizada segundo análise de fertilidade do solo e recomendação de Lamas
(2003). Na fundação foram utilizados 72 $\mathrm{g} \mathrm{cova}^{-1}$ de sulfato de amônia, $12 \mathrm{~g} \mathrm{cova}^{-1}$ de superfosfato simples e $16 \mathrm{~g} \mathrm{cova}^{-1} \mathrm{de}$ cloreto de potássio. A cada três meses foi feita adubação em cobertura, com 72 $\mathrm{g}$ touceira $^{-1}$ de sulfato de amônia e $16 \mathrm{~g}$ touceira $^{-1}$ de cloreto de potássio. Como suprimento de matéria orgânica foi aplicado trimestralmente esterco bovino curtido (13 $\mathrm{kg} \mathrm{m}^{-2}$ de touceira). O delineamento experimental foi blocos ao acaso, sendo avaliados 26 genótipos (Tabela 1), com quatro repetições. Este material, doado por produtores da região, faz parte da Coleção de Germoplasma de Helicônias da Universidade Federal Rural de Pernambuco (UFRPE).

Os rizomas foram lavados, as raízes cortadas e, em seguida, submetidos a tratamento fitossanitário com nematicida, inseticida e fungicida. $\mathrm{O}$ espaçamento de plantio foi de $1,5 \mathrm{~m}$ entre rizomas na linha e $3 \mathrm{~m}$ entre linhas. Foram plantados dois rizomas de cada genótipo por parcela em duas áreas experimentais (Tabela 1): Área 1 - com 21,0 m x 15,0 m, a pleno sol; Área 2 com 10,5 m x 15,0 m, a meia-sombra (tela de sombreamento de 50\%). A distribuição dos genótipos nas áreas a pleno sol ou a meia-sombra foi baseada nas observações das práticas de cultivos da Zona da Mata de Pernambuco. Os genótipos $H$. psittacorum x $H$. spathocircinata cultivar Golden Torch e $H$. bihai foram plantados nas duas áreas por serem espécies muito difundidas comercialmente.

Cinqüenta-e-quatro dias após o plantio (DAP) foi mantida na parcela apenas a touceira que apresentou melhor desenvolvimento e vigor. As avaliações do número total de perfilhos emitidos por touceira (NPT) foram iniciadas 59 DAP e realizadas, em média, a cada 29 dias. Os perfilhos foram etiquetados e numerados seguindo a ordem de emissão. As touceiras foram medidas trimestralmente com esquadro, sendo obtidos os valores $\mathrm{L}_{1}$ (lado entre linhas) e $\mathrm{L}_{2}$ (lado entre plantas), em centímetros. A área de ocupação da touceira (AOT) correspondeu ao produto da multiplicação de $\mathrm{L}_{1}$ por $\mathrm{L}_{2}\left(\mathrm{~cm}^{2}\right)$. A partir da AOT foi calculada a taxa de ocupação da parcela (TOP) (AOT/área da parcela, em \%).
As análises estatísticas foram efetuadas segundo o modelo matemático de Person, utilizando o programa SWNTIA (EMBRAPA, 1996). Os dados de NPT, previamente transformados em $\sqrt{ }(x+0,5)$ e os dados de AOT, transformados em Öx, foram submetidos à análise de variância. As médias foram agrupadas pelo teste de Scott-Knott (P $<0,05$ ) (Scott \& Knott, 1974). Adicionalmente foi realizada a análise de coeficiente de correlação simples entre as médias de NPT e de AOT dos genótipos cultivados a pleno sol e a meia sombra, ao nível de significância de 5\%. Foi utilizado o programa SAEG (1983).

\section{RESULTADOS E DISCUSSÃO}

Houve diferença significativa entre as médias dos genótipos a $1 \%$ de probabilidade pelo teste de $\mathrm{F}$ para número de perfilhos por touceira (NPT), avaliados 316 DAP, e para a área de ocupação da touceira (AOT) avaliada 304 DAP, o que demonstra haver variabilidade entre os genótipos para estas características.

Considerando todos os genótipos cultivados a pleno sol, foram formadas três classes significativamente distintas (Scott-Knott, $\mathrm{P}<0,05)$ para NPT. $H$. psittacorum cultivar Red Opal apresentou a maior média para NPT $(57,0)$ constituindo isoladamente a classe I. As classes II e III apresentaram médias para NPT variando de 32,7 a 42,5 e de 18,3 a 30,3 , respectivamente. É interessante destacar que, nesta avaliação, os sete genótipos das cultivares e híbridos de $H$. psittacorum foram distribuídos em todas as classes. A cultivar Red Opal, conforme mencionado acima, agrupouse na classe I; as cultivares Suriname Sassy, Golden Torch Adrian, Alan Carle, Strawberries \& Cream e Golden Torch agruparam-se na classe II; e, a cultivar Red Gold, na classe III. Os genótipos da espécie $H$. latispatha também foram distribuídos em classes distintas para NPT: na classe II a cultivar Distans e, na classe III, a cultivar Red-Yellow Gyro.

Os genótipos cultivados a meia-sombra se distribuíram em três classes distintas (Scott-Knott, $\mathrm{P}<0,05)$. A classe I apresentou apenas genótipo $H$. psittacorum x $H$. spathocircinata cultivar Golden Torch, que apresentou a 
Tabela 1. Características de genótipos da Coleção de Germoplasma de Helicônias da Universidade Federal Rural de Pernambuco. Camaragibe, UFRPE, 2004.

\begin{tabular}{|c|c|c|c|c|}
\hline Genótipos* & Área** & $\begin{array}{l}\text { Porte da } \\
\text { Planta*** }^{*}\end{array}$ & $\begin{array}{l}\text { Posição da } \\
\text { inflorescência }\end{array}$ & $\begin{array}{l}\text { Cor predominante } \\
\text { das brácteas }\end{array}$ \\
\hline H. bihai (L.) L. cultivar Nappi Yellow & 2 & Grande & Ereta & Vermelho-Amarelo \\
\hline H. bihai (L.) L. & 1 e 2 & Grande & Ereta & Vermelho \\
\hline H. bihai (L.) L. cultivar Kamehameha & 1 & Grande & Ereta & Vermelho-Amarelo \\
\hline H. caribaea Lamark x H. bihai (L.) L. cultivar Carib Flame & 2 & Grande & Pendente & Vermelho \\
\hline H. collinsiana Griggs & 2 & Grande & Pendente & Vermelho \\
\hline H. episcopalis Vellozo & 2 & Médio & Ereta & Laranja-Amarelo \\
\hline H. Iatispatha Bentham cultivar Distans & 1 & Médio & Ereta & Vermelho-Verde \\
\hline H. latispatha Bentham cultivar Red-Yellow Gyro & 1 & Médio & Ereta & Laranja \\
\hline H. orthotricha L. Anderss & 1 & Médio & Ereta & Vermelho-Laranja \\
\hline H. pendula Wawra & 2 & Grande & Pendente & Vermelho \\
\hline H. psittacorum L.f. cultivar Red Gold & 1 & Pequeno & Ereta & Amarelo-Laranja \\
\hline H. psittacorum L.f. x H. spathocircinata Aristeguieta cultivar Golden Torch & 1 e 2 & Pequeno & Ereta & Amarelo \\
\hline H. psittacorum L.f. cultivar Red Opal & 1 & Pequeno & Ereta & Laranja \\
\hline H. psittacorum L.f. cultivar Strawberries \& Cream & 1 & Pequeno & Ereta & Rosa - Amarelo \\
\hline H. psittacorum L.f. cultivar Suriname Sassy & 1 & Pequeno & Ereta & Rosa-Verde \\
\hline H. psittacorum L.f. x H. spathocircinata Aristeguieta cultivar Alan Carle & 1 & Pequeno & Ereta & Amarelo- Laranja \\
\hline H. psittacorum L.f. x H. spathocircinata Aristeguieta cultivar Golden Torch Adrian & 1 & Pequeno & Ereta & Amarelo - Vermelho \\
\hline H. rauliniana Barreiros & 2 & Grande & Pendente & Vermelho \\
\hline H. rostrata Ruiz \& Pávon (03 dias pós-colheita) & 1 & Médio & Pendente & Vermelho \\
\hline H. rostrata Ruiz \& Pavón (10 dias pós-colheita) & 1 & Médio & Pendente & Vermelho \\
\hline H. rostrata Ruiz \& Pávon & 2 & Médio & Pendente & Vermelho \\
\hline H. stricta Huber cultivar Fire Bird & 2 & Grande & Ereta & Vermelho \\
\hline H. stricta Huber & 2 & Grande & Ereta & Laranja \\
\hline H. wagneriana Peters & 1 & Grande & Ereta & Amarelo-Laranja \\
\hline H. pseudoaemygdiana (L.) Emygdioie. \& E. Santos & 1 & Médio & Ereta & Amarelo \\
\hline Heliconia x nickeriensis Maas \& deRooij & 1 & Pequeno & Ereta & Laranja-Amarelo \\
\hline
\end{tabular}

"Identificação baseada em Berry e Kress (1991); ${ }^{* *}$ Área 1-pleno sol; Área 2-meia sombra (tela de sombreamento de 50\%).***Classificação quanto ao porte: pequeno (altura inferior a 1,50 m); médio (altura entre 1,51 m a 2,50 m) e grande (altura superior a 2,51 m).

maior média para NPT (43,7). A classe II foi formada por apenas três genótipos, com limites para NPT de 28,8 a 34,0. A classe III agregou seis genótipos, com limite inferior de 11,7 e superior de 25,3 perfilhos por touceira.

Em relação à área de ocupação da parcela (AOT), os genótipos cultivados a pleno sol formaram quatro classes estatisticamente distintas (Scott-Knott, $\mathrm{P}<0,05)$, o que mostra uma maior variação entre os mesmos quanto à área de ocupação da touceira após dez meses. Os genótipos Heliconia x nickeriensis que atingiu $22.541 \mathrm{~cm}^{2}$ (taxa de ocupação da parcela superior a $100 \%$ ) e $H$. psittacorum cultivar Strawberries \& Cream com 17.556 $\mathrm{cm}^{2}$ (taxa de ocupação da parcela superior a 78,03\%), formaram a classe I. A classe II, com AOT variando entre 7.102 a $13.754 \mathrm{~cm}^{2}$ foi composta pelas cultivares de $H$. psittacorum, Golden Torch, Red Opal, Suriname Sassy, alem de $H$. latispatha cultivar Red-Yellow Gyro. As classes III e IV reuniram os genótipos com AOT entre 5.076 (H. latispatha cultivar Distans) e $2.864 \mathrm{~cm}^{2}$ (H. rostrata) e 725 (H. orthotricha) e $2.347 \mathrm{~cm}^{2}(H$. psittacorum cultivar Red Gold), respectivamente.

No experimento conduzido a meiasombra, nenhum dos genótipos apresentou AOT superior a $10.201 \mathrm{~cm}^{2}(45,34 \%$ da área total da parcela), formando duas classes distintas (Scott-Knott, $\mathrm{P}<0,05$ ): a classe I, com AOT entre 7.276 e 10.201 , composta por apenas três genótipos (H. stricta cultivar Fire Bird, H. psittacorum cultivar Golden Torch e H. stricta) e a classe II, com AOT entre 750 e 3.936, composta por sete genótipos.

De modo geral, observou-se que as helicônias de pequeno porte como, por exemplo, as cultivares e híbridos de $H$. psittacorum perfilharam mais que as helicônias de grande porte, em ambas as condições de plantio. Os valores de NPT e AOT da $H$. psittacorum x $H$. spathocircinata cultivar Golden Torch e H. bihai cultivadas a pleno sol e a meia-sombra foram semelhantes, indicando não ser necessário o plantio com sombreamento.

As correlações entre o NPT e a AOT, a pleno sol $\left(\mathrm{r}=0,53^{* *}\right)$ e a meia sombra $\left(\mathrm{r}=0,71^{* *}\right)$, indicam que, de modo geral, os genótipos que perfilham muito, apresentam expansão mais rápida da touceira, sendo definidos como de crescimento aberto (Criley, 1988). Estes devem ser plantados em espaçamentos maiores que 1,5 x 3,0 m, caso contrário será necessário desbaste de perfilhos, para que não haja invasão nas entrelinhas. Espaçamentos reduzidos acarretam o rápido adensamento das plantas necessitando renovar o plantio em intervalos de tempo menores. 
Quanto aos genótipos que apresentaram reduzido perfilhamento e expansão lenta da touceira, definidos por Criley (1988) como de crescimento agrupado, não é recomendado espaçamento menor que o adotado devido às plantas apresentarem porte superior a $1,51 \mathrm{~m}$. Dessa forma, evitar-se-ia a concorrência entre os perfilhos por luz e, conseqüentemente, a redução da produção (Fernandes, 2000) e da qualidade das inflorescências (Ibiapaba et al., 1997).

Genótipos de grande porte com inflorescências eretas e crescimento agrupado como, por exemplo $H$. bihai, mostraram tendência ao tombamento da planta, levando à formação de inflorescências inclinadas em relação à haste, característica indesejável para a comercialização devido à dificuldade de embalagem e transporte.

Embora o número de perfilhos por touceira e a área de ocupação da touceira tenham sido avaliados somente até 316 DAP, observou-se claramente que a adoção de um espaçamento único para helicônia é inadequado. Este experimento continua sendo conduzido para avaliação e caracterização das inflorescências, pois pretende-se observar o perfilhamento, a expansão, a produção da touceira e a qualidade das inflorescências.

\section{AGRADECIMENTOS}

Os autores agradecem à CAPES, FUNDECI - ETENE e ao PROMATA pelo suporte financeiro; à Maria do Carmo Ferraz Teixeira e produtores da RECIFLORA, pela doação dos rizomas, e a todos da Fazenda Bem-Te-Vi e do Laboratório de Floricultura da UFRPE, onde foram realizadas as atividades.

\section{REFERÊNCIAS}

AKI A; PEROSA JM. 2002. Aspectos da produção e consumo de flores e plantas ornamentais no Brasil. Revista Brasileira de Horticultura Ornamental 8: 13-23.

ATEHORTUA L. 1998. Aves del paraiso Strelitzia Gingers Alpinia Heliconia Medellín: Universidad de Antioquia 66p.

BERRY F; KRESS WJ. 1991. Heliconia: an identification guide. Washington and London: Smithsonian Institution Press. 334p.

CASTRO CEF de. 1995. Helicônia para exportação: aspectos técnicas da produção. Brasília: MAARA-SDR-FRUPEX/SPI. $44 \mathrm{p}$. (FRUPEX-Publicações Técnicas).

CATLEY JL; BROOKING IR. 1996. Temperature and light influence growth and flower production in Heliconia Golden Torch. HortScience 31: 213-217.

CRILEY RA. 1988. Propagation methods for gingers and heliconias. Bulletin Heliconia Society International 2: 6-7.

CRILEY RA. 1989. Development of Heliconia and Alpinia in Hawaii: cultivar selection and culture. Acta Horticulturae 246: 247-258.

CRILEY RA; BROSCHAT TK. 1992. Heliconia: botany and horticulture of new floral crop. Horticulturae Review 14: 1-55.

EMBRAPA. Centro Nacional de Pesquisa Tecnológica em Informática para a Agricultura. 1996. SWNTIA, versão 4.2.1. Instalação e programa. Campinas: EMBRAPA-CNPTIA (Disquete).

FERNANDES EP. 2000. Crescimento e produção de Heliconia psittacorum L. em função de adubação mineral e densidade de plantio. Goiânia: UFG. 99p. (Dissertação mestrado).
GEERTSEN V. 1989. Influence of photoperiod and temperature on the growth and flower production of Heliconia psittacorum 'Tay'. Acta Horticulturae 252: 117-122.

IBIAPABA MVB da; LUZ JMQ; INNECCO, R. 1997. Comportamento de duas espécies de helicônia em diferentes espaçamentos de plantio em Fortaleza (CE). Revista Brasileira de Horticultura Ornamental 3: 74-79.

ITEP-Instituto de Tecnologia de Pernambuco. 2005, 09 de janeiro. Disponível em http:// www.itep.br/lamepe.ASP.

JUNQUEIRA AH; PEETZ MS. 2002. Os pólos de produção de flores e plantas ornamentais do Brasil: uma análise do potencial exportado. Revista Brasileira de Horticultura Ornamental 8: 25-47.

KIYUNA I; ÂNGELO JA; COELHO PJ. 2004, 30 de setembro. Comportamento do comércio exterior brasileiro no primeiro trimestre de 2004. Disponível em http://www.iea.sp.gov.br/ out/verTexto.php?codTexto $=1420$

LAMAS AM. 2003. Floricultura Tropical-Avanços Tecnológicos. Fortaleza: Instituto Frutal (CD-ROM).

PAIVA WO de. 1998. A cultura de helicônias. Fortaleza: EMBRAPA-CNPAT. 20p. (Circular Técnica 2).

RIBEIRO TR; LOPES GGO; VIANA FD. 2002. Produção de mudas e flores de plantas ornamentais tropicais. Petrolina: EMBRAPA CPATSA, 41p. (Circular Técnica 2).

SAEG-Sistema para análises estatísticas e genéticas. 1983. Viçosa: Fundação Arthur Bernardes (Versão 5.0).

SCOTT AJ; KNOTT M. 1974. A cluster analysis method for grouping means in the analysis of variance. Biometrics 30: 507-512. 